\title{
Towards Self-configuration in Autonomic Electronic Institutions
}

\author{
Eva Bou ${ }^{1}$, Maite López-Sánchez ${ }^{2}$, and J.A. Rodríguez-Aguilar ${ }^{1}$ \\ ${ }^{1}$ IIIA - CSIC Artificial Intelligence Research Institute, Campus UAB 08193 \\ Bellaterra, Spain \\ $\{$ ebm,jar\}@iiia.csic.es \\ ${ }^{2}$ WAI, Volume Visualization and Artificial Intelligence, MAiA Dept., Universitat de \\ Barcelona \\ maite@maia.ub.es
}

\begin{abstract}
Electronic institutions (EIs) have been proposed as a means of regulating open agent societies. EIs define the rules of the game in agent societies by fixing what agents are permitted and forbidden to do and under what circumstances. And yet, there is the need for EIs to adapt their regulations to comply with their goals despite coping with varying populations of self-interested agents. In this paper we focus on the extension of EIs with autonomic capabilities to allow them to yield a dynamical answer to changing circumstances through the adaptation of their norms.
\end{abstract}

\section{Introduction}

The growing complexity of advanced information systems in the recent years, characterized by being distributed, open and dynamical, has given rise to interest in the development of systems capable of self-management. Such systems are known as self-* systems [1, where the ${ }^{*}$ sign indicates a variety of properties: self-organization, self-configuration, self-diagnosis, self-repair, etc. A particular approximation to the construction of self-* systems is represented by the vision of autonomic computing [2, which constitutes an approximation to computing systems with a minimal human interference. Some of the many characteristics of autonomic systems are: it must configure and reconfigure itself automatically under changing (and unpredictable) conditions; it must aim at optimizing its inner workings, monitoring its components and adjusting its processings in order to achieve its goals; it must be able to diagnose the causes of its eventual malfunctions and reparate itself; it must act in accordance to and operate into a heterogeneous and open environment.

In what follows we argue that are EIs 3 a particular type of self-* system. When looking at computer-mediated interactions we regard Electronic Institutions (EI) as regulated virtual environments wherein the relevant interactions among participating agents take place. EIs have proved to be valuable to develop open agent systems [4. However, the challenges of building open systems 
are still considerable, not only because of the inherent complexity involved in having adequate interoperation of heterogeneous agents, but also because the need for adapting regulations to comply with institutional goals despite varying agents' behaviors. Particularly, when dealing with self-interested agents.

The main goal of this work consists in studying how to endow an EI with autonomic capabilities that allow it to yield a dynamical answer to changing circumstances through the adaptation of its regulations. Among all the characteristics that define an autonomic system we will focus on the study of self-configuration as pointed out in 2] as a second characteristic: "An autonomic computing system must configure and reconfigure itself under varying (and in the future, even unpredictable) conditions. System configuration or "setup" must occur automatically, as well as dynamic adjustments to that configuration to best handle changing environments".

The paper is organized as follows. In section 2 we introduce the notion of autonomic electronic institution as an extension of the classic notion of electronic institution along with a general model for norm adaptation. Section 3 details a case study to be employed as a scenario wherein to test the model presented in section 2, Section 4 provides some preliminary, empirical results. Finally, section 5] summarizes some conclusions and outlines paths to future research.

\section{Autonomic Electronic Institutions}

The idea behind EIs [5] is to mirror the role traditional institutions play in the establishment of "the rules of the game"-a set of conventions that articulate agents' interactions- but in our case applied to agents (be them human or software) that interact through messages whose (socially relevant) effects are known to interacting parties. The essential roles EIs play are both descriptive and prescriptive: the institution makes the conventions explicit to participants, and it warrants their compliance. EIs involve a conceptual framework to describe agent interactions as well as an engineering framework [ 6 to specify and deploy actual interaction environments.

Although EIs can be regarded as the computational counterpart of human institutions for open agent systems, there are several aspects in which they are nowadays lacking. According to North 7 human institutions are not static; they may evolve over time by altering, eliminating or incorporating norms. In this way, institutions can adapt to societal changes. Nonetheless, neither the current notion of EI in [3] nor the engineering framework in [6] support norm adaptation so that an EI can self-configure. Thus, in what follows we study how to extend the current notion of EI in [3] to support self-configuration.

First of all, notice that in order for norms to adapt, we believe that a "rational" view of EIs must be adopted (likewise the rational view of organizations in [8]) and thus consider that EIs seek specific goals. Hence, EIs continuously adapt their norms to fulfill their goals. Furthermore, we assume that an EI is situated in some environment that may be either totally or partially observable by the EI and its participating agents. 
With this in mind, we observe that according to [3] an EI is solely composed of: a dialogic framework establishing the common language and ontology to be employed by participating agents; a performative structure defining its activities along with their relationships; and a set of norms defining the consequences of agents' actions. From this follows that further elements are required in order to incorporate the fundamental notions of goal and norm transition as captured by the following definition of autonomic electronic institution.

Definition 1. Given a finite set of agents A, we define an Autonomic Electronic Institution (AEI) as a tuple $\left\langle P S, N, D F, G, P_{i}, P_{e}, P_{a}, V, \delta\right\rangle$ where:

- PS stands for a performative structure;

- $N$ stands for a finite set of norms;

- DF stands for a dialogic framework;

- $G$ stands for a finite set of institutional goals;

- $P_{i}=\left\langle i_{1}, \ldots, i_{s}\right\rangle$ stands for the values of a finite set of institutional properties, where $i_{j} \in \mathbb{R}, 1 \leq j \leq s$ contains the value of the $j$-th property;

$-P_{e}=\left\langle e_{1}, \ldots, e_{r}\right\rangle$ stands for the values of the environment properties, where each $e_{j}$ is a vector, $e_{j} \in \mathbb{R}^{n_{j}} 1 \leq j \leq r$ contains the value of the $j$-th property;

$-P_{a}=\left\langle a_{1}, \ldots, a_{n}\right\rangle$ stands for the values that characterize the institutional state of the agents in $A$, where $a_{j}=\left\langle a_{j_{1}}, \ldots, a_{j_{m}}\right\rangle \quad 1 \leq j \leq n$ stands for the institutional state of agent $A_{j}$;

- $V$ stands for a finite set of reference values; and

- $\delta: P S \times N \times G \times V \rightarrow P S \times N$ stands for a normative transition function that maps a performative structure and a set of norms into a new performative structure and a new set of norms given a set of goals and a set of values for the reference variables.

Notice that a major challenge in the design of an AEI is to learn a normative transition function, $\delta$, that ensures the achievement of its institutional goals under changing conditions. Next, we dissect the new elements composing an AEI.

An AEI employs norms to constrain agents' behaviors and to assess the consequences of their actions within the scope of the institution. Although there is a plethora of formalizations of the notion of norm in the literature, in this paper we adhere to a simple definition of norms as effect propositions as defined in [9]:

Definition 2. An effect proposition is an expression of the form

$$
A \text { causes } F \text { if } P_{1}, \ldots, P_{n}
$$

Where $A$ is an action name, and each of $F, P_{1}, \ldots, P_{n}(n \geq 0)$ is a fluent expression. About this proposition we say that it describes the effect of $A$ on $F$, and that $P_{1}, \ldots, P_{n}$ are its preconditions. If $\mathrm{n}=0$, we will drop if and write simply $A$ causes $F$. From this definition, changing a norm amounts to changing either its pre-conditions, or its effect(s), or both. 
Agents participating in an AEI have their social interactions mediated by the institution according to its norms. As a consequence of his interactions, only the institutional (social) state of an agent can change since an AEI has no access whatsoever to the inner state of any participating agent. Therefore, given a finite set of participating agents $A=\left\{A_{1}, \ldots, A_{n}\right\}$ where $n \in \mathbb{N}$, each agent $A_{i} \in A$ can be fully characterized by his institutional state, represented as a tuple of observable values $\left\langle a_{i_{1}}, \ldots, a_{i_{m}}\right\rangle$ where $a_{i_{j}} \in \mathbb{R} 1 \leq j \leq m$. Thus, the actions of an agent within an AEI may change his institutional state according to the institutional norms.

The main objective of an AEI is to accomplish its goals. For this purpose, and AEI will adapt its norms. We assume that the institution can observe the environment, the institutional state of the agents participating in the institution, and its own state to assess whether its goals are accomplished or not. Thus, from the observation of environment properties $\left(P_{e}\right)$, institutional properties $\left(P_{i}\right)$, and agents' institutional properties $\left(P_{a}\right)$, an AEI obtains the reference values required to determine the fulfillment of goals. Formally, the reference values are defined as a vector $V=\left\langle v_{1}, \ldots, v_{q}\right\rangle$ where each $v_{j}$ results from applying a function $h_{j}$ upon the agents' properties, the environmental properties and/or the institutional properties; $v_{j}=h_{j}\left(P_{a}, P_{e}, P_{i}\right), 1 \leq j \leq q$.

Finally, we can turn our attention to institutional goals. An example of institutional goal for the Traffic Regulation Authority could be to keep the number of accidents below a given threshold. In other words, to ensure that a reference values satisfies some constraint.

Formally we define the goals of an AEI as a finite set of constraints $G=$ $\left\{c_{1}, \ldots, c_{p}\right\}$ where each $c_{i}$ is defined as an expression $g_{i}(V) \triangleleft\left[m_{i}, M_{i}\right]$ where $m_{i}, M_{i} \in \mathbb{R}, \triangleleft$ stands for either $\in$ or $\notin$, and $g_{i}$ is a function over the reference values. In this manner, each goal is a constraint upon the reference values where each pair $m_{i}$ and $M_{i}$ defines an interval associated to the constraint. Thus, the institution achieves its goals if all $g_{i}(V)$ values satisfy their corresponding constraints of being within (or not) their associated intervals.

\subsection{Norm Adaptation}

A major challenge in the design of an AEI is to learn a normative transition function that allows to accomplish institutional goals under changing situations. In this work, we concentrate on norm adaptation and therefore we consider that there is no definition of performative structure. Thus, institutional goals must be accomplished through norms, which will be the only means of regulating agents' actions. We are considering the normative transition function defined in 1 in a more simple way, $\delta: N \times G \times V \rightarrow N$, namely as a normative transition function that maps a set of norms into a new set of norms.

From the definition 2 of norm, changing a norm amounts to changing either its pre-conditions, or its effects, or both. Norms can be parameterized, and therefore we propose that each norm, $N_{i} \in N$, has a set of parameters $\left\langle p_{i_{1}}, \ldots, p_{i_{m}}\right\rangle \in \mathbb{R}^{i_{m}}$. Notice that when the parameters of the norms are associated to the pre-conditions and/or to the effects, changing the values of these 
parameters means changing the norm. When we refer to change the norms or to adapt the norms we are referring to change or to adapt the values of the parameters of the norms. Norms have associated parametres that can be changed to increase its persuasiveness depending on the agent population behavior. We propose to learn the normative transition function by exploring the space of parameter values in search for the ones that best accomplish goals for a given population of agents. In this manner, if we can automatically adapt norms to the global behavior of an agent population, then, we can repeat it for a number of different agent populations and thus characterize the overall normative transition function.

Figure 1 describes how this learning process is performed for a given population of agents $(A)$ using an evolutionary approach. We have an initial set of individuals $\left(\left\langle I_{1}, . ., I_{n}\right\rangle\right)$, where each individual represents a set of norm parameters $\left(\left\{\left\langle p_{11}, . ., p_{1 m}\right\rangle, \ldots,\left\langle p_{i 1}, . ., p_{i m}\right\rangle\right\}\right)$. The institution performs a simulation for each individual with the population of agents $A$, so that the norms represented by each individual can be evaluated according to the institutional goals (Norm evaluation).Finally, the AEI compiles the evaluations of all individuals in order to perform the Norm adaptation process that results with a new set of individuals (New norms) to be used as an initial set of individuals for next step in the learning process.

Since we are working with a complex system, we propose use an evolutionary approach for learning due to the fact that the institutional objective function can be naturally mapped to the fitness function and an evolutionary approach provides a solution good enough. Notice that the AEI does not learn any agent parameter, it learns the best parameters by simulation for a certain population of agents, that is whose values will be changed by the normative transition function and by the PS transition function. It is a first step learning where the AEI learns by simulation the best parameters for a list of populations, thus, in a next step the AEI could use this learning in a real environment to adapt itself to any population of agents (e.g., using Case-Based Reasoning (CBR) problem solving technique).

\section{Case Study: Traffic Control}

Traffic control is a well-known problem that has been approached from different perspectives, which range from macro simulation for road net design [10] to traffic flow improvement by means of multi-agent systems [11. We tackle this problem from the Electronic Institutions point of view, and therefore, this section is devoted to specify how traffic control can be mapped into Autonomic Electronic Institutions.

In this manner, we consider the Traffic Regulation Authority as an Autonomic Electronic Institution, and cars moving along the road network as agents inside the institution. Considering this set-up, traffic norms regulated by Traffic Authorities can therefore be translated in a straight forward manner into norms belonging to the Electronic Institution. Norms within this normative environment 


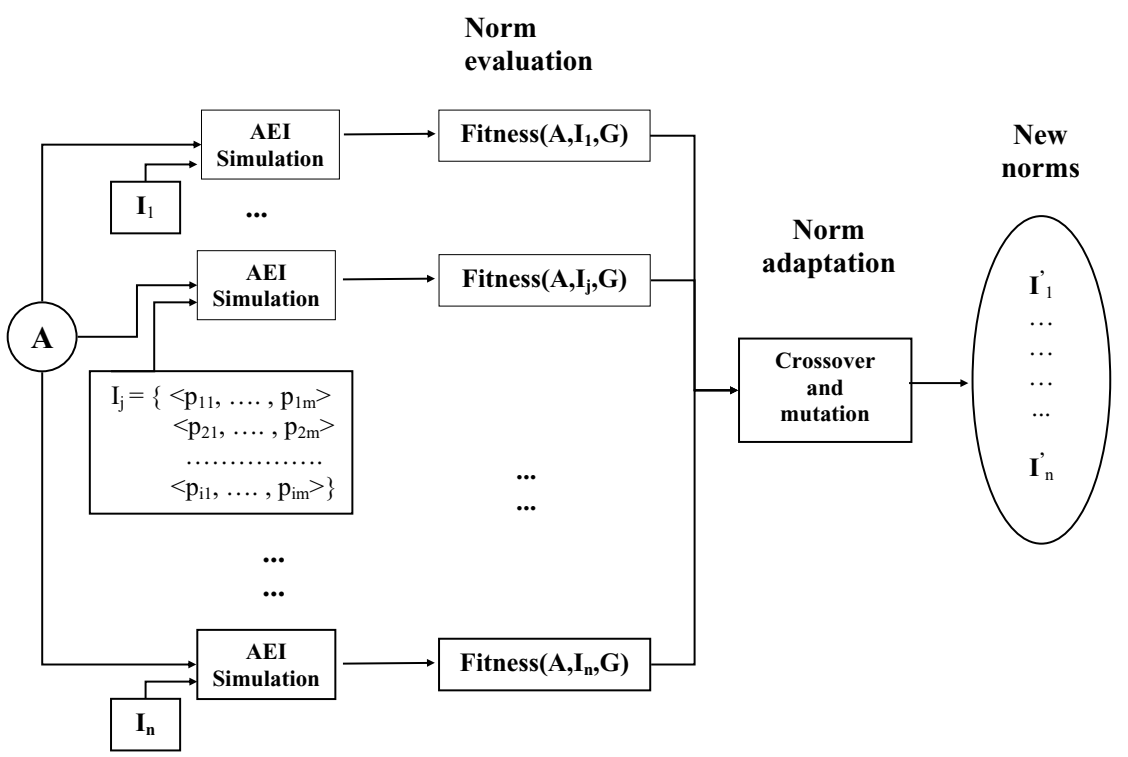

Fig. 1. Example of an step in norm adaptation using an evolutionary approach

are thus related to actions performed by cars (in fact, in our case, they are always restricted to that). Additionally, norms do have associated penalties that are imposed to those cars refusing or failing to follow them. In our case study, we assume that the Traffic Authority is always aware of norm violations: cars may or may not respect rules, but they are not able to avoid the consequences of their application. Furthermore, our Electronic Institution is able to change norms based on its goals - just as traffic authorities do modify their traffic rules- and, therefore, it is considered to be autonomic.

Our AEI sets up a normative environment where cars do have a limited amount of credit (just as some real world driving license credit systems) so that norm offenses cause credit reductions. The number of points subtracted for each traffic norm violation is specified by the sanction associated to each norm, and this sanction can be changed by the regulation authority (that is, our AEI) if its change leads -or contributes to- the accomplishment of goals. Eventually, those cars without any remaining points are forbidden to circulate. On the other hand, we assume a non-closed world, so expelled cars are replaced by new ones having the total amount of points.

Getting into more detail, we focus on a two-road junction. It is a very restrictive problem setting, but it is complex enough to allow us to tackle the problem without losing control of all the factors that may influence the results. In particular, no traffic signals (neither yield or stop signals nor traffic lights) are considered, therefore, cars must only coordinate by following the traffic norms imposed by the AEI. Our institution is required to define these traffic norms based on general goals such as minimization of the number of accidents or deadlock avoidance. 


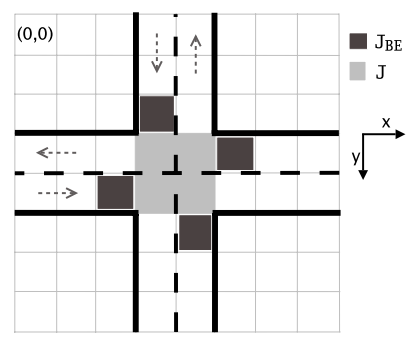

Fig. 2. Grid environment representation of a 2-lane road junction

We model the environment as a grid composed by road and field cells. Road cells define 2 orthogonal roads that intersect in the center (see figure 2). Discretization granularity is such that cars have the size of a cell. As section 3.2 details, our model has been developed with the Simma tool [12. Although the number of road lanes can be changed parametrically, henceforth we assume the 2lane case. Next subsections are devoted to define this "toy problem" and present our solution proposal in terms of it. But before that, we introduce some nomenclature definitions:

- $A_{i}$ : an agent $i$, agents correspond to cars.

- $t$ : time step. Our model considers discrete time steps (ticks).

- $\left(J_{x}, J_{y}\right)$ : size in $x, y$ of our road junction area.

- $J$ : inner road junction area with $\left(x_{0}^{J}, y_{0}^{J}\right)$ as top left cell inside it $J=\left\{(x, y) \mid x \in\left[x_{0}^{J}, x_{0}^{J}+J_{x}-1\right], y \in\left[y_{0}^{J}, y_{0}^{J}+J_{y}-1\right]\right\}$

Considering the $4 \mathrm{~J}$ cells in the junction area of Figure 2, $J=\left\{\left(x_{0}^{J}, y_{0}^{J}\right),\left(x_{0}^{J}+1, y_{0}^{J}\right),\left(x_{0}^{J}, y_{0}^{J}+1\right),\left(x_{0}^{J}+1, y_{0}^{J}+1\right)\right\}$.

- $J_{B E}$ : Junction Boundary Entrance, set of cells surrounding the junction that can be used by cars to access it. They correspond to cells near by the junction that belong to incoming lanes. Figure 2 depicts $J_{B E}=\left\{\left(x_{0}^{J}, y_{0}^{J}-1\right),\left(x_{0}^{J}-\right.\right.$ $\left.\left.1, y_{0}^{J}+J_{y}-1\right),\left(x_{0}^{J}+J_{x}-1, y_{0}^{J}+J_{y},\left(x_{0}^{J}+J_{x}, y_{0}^{J}\right)\right)\right\}$.

Nevertheless, the concept of boundary is not restricted to adjacent cells: a car can be also considered to be coming into the junction if it is located one -or even a few-cells away from the junction.

- $\left(x_{i}^{t}, y_{i}^{t}\right)$ : position of car $A_{i}$ at time $\mathrm{t}$, where $(x, y) \in \mathbb{N} \times \mathbb{N}$ stands for a cell in the grid.

- $\left(h_{i x}^{t}, h_{i y}^{t}\right)$ : heading of car $A_{i}$, which is located in $(x, y)$ at time t. Heading directions run along $x, y$ axes and are considered to be positive when the car moves right or down respectively. In our orthogonal environment, heading values are: 1 if moving right or down; -1 if left or up; and 0 otherwise (i.e., the car is not driving in the axe direction). In this manner, car4's heading on the right road of figure 3 is $(-1,0)$.

\subsection{AEI Specification}

Environment. As mentioned above, we consider the environment to be a grid. This grid is composed of cells, which can represent roads or fields. The main 
difference among these two types is that road cells can contain cars. Indeed, cars move among road cells along time.

Figure 2 depicts a $8 \times 8$ grid example. The top left corner of the grid represents the origin in the $\mathrm{x}, \mathrm{y}$ axes. Thus, in the example, cell positions range from $(0,0)$ in the origin up to $(7,7)$ at the bottom-right corner. Additionally, a cell is a road if one of its $\mathrm{x}, \mathrm{y}$ coordinates belong to $J$ inner junction area (see previous definition).

We define this grid environment as:

$$
\begin{gathered}
P_{e}=\left\langle\left(x, y, \alpha, r, d_{x}, d_{y}\right)\right| 0 \leq x \leq \max _{x}, 0 \leq y \leq \max _{y}, \\
\left.\alpha \subseteq P(A), r \in[0,1], d_{x} \in[-1,0,1], d_{y} \in[-1,0,1]\right\rangle
\end{gathered}
$$

being $x$ and $y$ the cell position, $\alpha$ defines the set of agents inside the grid cell $(x, y), \mathrm{r}$ indicates whether this cell represents a road or not, and, in case it is a road, $d_{x}$ and $d_{y}$ stand for the lane direction, whose values are the same as the ones for car headings. Noticie that the institution can observe the environment properties along time, we use $P_{e}^{t}$ to refer the values of the grid environment at a specific time t. This discretized environment can be observed both by the institution and cars. The institution observes and keeps track of its evolution along time, whilst cars do have locality restrictions on their observations.

Agents. We consider $A=\left\langle A_{1}, \ldots, A_{n}\right\rangle$ to be a finite set of $n$ agents in the institution. As mentioned before, agents correspond to cars that move inside the grid environment, with the restriction that they can only move within road cells. Additionally, agents are given an account of points which decreases with traffic offenses. The institution forbids agents to drive without points in their accounts. The institution can observe the $P_{a}=\left\langle a_{1}, \ldots, a_{n}\right\rangle$ agents' institutional properties, where

$$
a_{i}=\left\langle x_{i}, y_{i}, h_{i x}, h_{i y}, \text { speed }_{i}, \text { indicator }_{i}, \text { of fenses }_{i},\right.
$$

These properties stand for: car $A_{i}$ 's position within the grid, its heading, its speed, whether the car is indicating a trajectory change for the next time step (that is, if it has the intention to turn, to stop or to move backwards), the norms being currently violated by $A_{i}$, wether the car is involved in an accident, the distance between the car and the car ahead of it; and, finally, agent $A_{i}$ 's point account. Notice that the institution can observe the agent properties along time, we use $a_{i}^{t}$ to refer the agent $A_{i}$ 's properties at a specific time t.

Reference values. In addition to car properties, the institution is able to extract reference values from the observable properties of the environment, the participating agents and the institution. Thus, these reference values are computed as a compound of other observed values. Considering our road junction case study, we identity different reference values:

$$
\begin{gathered}
V=\langle\text { num_collisions, num_crashed, num_offenses }, \\
\text { num_blocked }\rangle
\end{gathered}
$$


where num_collisions indicates total number of collisions for last $t_{w}$ ticks $(0 \leq$ $\left.t_{w} \leq t_{\text {now }}\right)$ :

$$
\text { num_collisions }=\sum_{t=t_{\text {now }}-t_{w}}^{t_{\text {now }}} \sum_{e \in P_{e}^{t}} f\left(e_{\alpha^{t}}\right)
$$

being $P_{e}^{t}$ the values of the grid environment at time t, $e_{\alpha^{t}}$ the $\alpha^{t}$ component of element $e \in P_{e}^{t}$ and

$$
f\left(e_{\alpha^{t}}\right)=\left\{\begin{array}{ll}
1 & \text { if }\left|e_{\alpha^{t}}\right|>1 \\
0 & \text { otherwise }
\end{array} .\right.
$$

Furthermore, num_crashed counts the number of cars involved in accidents for last $t_{w}$ ticks, num_of fenses indicates the total number of offenses accumulated by all agents during last $t_{w}$ ticks, and finally, num_blocked shows how many cars have been blocked by other cars for last $t_{w}$ ticks.

Goals. Goals are institutional goals. The aim of the traffic authority institution is to accomplish as many goals as possible. The institution tries to accomplish these goals by defining a set of norms (see subsection 3.1).

Institutional goals are defined as constraints upon a combination of reference values. Considering our scenario, we define restrictions as intervals of acceptable values for the previous defined reference values $(V)$ so that we consider the institution accomplishes its goals if $V$ values are within their corresponding intervals. In fact, the aim is to minimize the number of accidents, the number of traffic offenses, as well as the number of blocked cars by establishing the list of institutional goals $G$ as:

$G=\langle$ num_collisions $\in[0$, MaxCollisions $]$, num_crashed $\in[0$, MaxCrashed $]$, num_offenses $\in[0$, MaxOffenses $]$, num_blocked $\in[0$, MaxBlocked $]\rangle$

Norms. Autonomic Electronic Institutions try to accomplish goals by defining norms. Norms have associated penalties that are imposed to those cars refusing or failing to follow them. These penalties can be parameterized to increase its persuasiveness depending on the agent population behavior.

Considering a road junction without traffic signals, priorities become basic to avoid collisions. We consider, as in most continental Europe, that the default priority is to give way to the right. This norm prevents a car $A_{i}$ located on the Junction Boundary Entrance $\left(J_{B E}\right)$ to move forward or to turn left whenever there is another car $A_{j}$ on its right. For example, car 1 in figure 3 must wait for car 2 on its right, which must also wait for car 3 at the bottom $J_{B E}$. The formalization in table 1 can be read as follows: "if car $A_{i}$ moves from a position in $J_{B E}$ at time $t-1$ to its next heading position at time $t$ without indicating a right turn, and if it performs this action when having a car $A_{j}$ at the $J_{B E}$ on its right, then the institution will fine $A_{i}$ by decreasing its points by a certain amount" (see figure 4).

Where the predicate $\operatorname{in}\left(a_{i}\right.$, Region, $\left.t\right)$ in table 1 is equivalent to $\exists\left(x, y, \alpha^{t}, r, d_{x}, d_{y}\right) \in E^{t}$ so that $(x, y) \in$ Region and $a_{i} \in \alpha^{t}$ and $\operatorname{right}\left(a_{i}, a_{j}, t\right)$ is a boolean function that returns true if car $a_{j}$ is located at $J_{B E}$ area on the right side of car $a_{i}$. For the 2-lane $J_{B E}$ case in Figure 2 it corresponds to the formula: $\left(x_{i}^{t}-h_{i y}^{t}+h_{i x}^{t} J_{x}, y_{i}^{t}+h_{i x}^{t}+h_{i y}^{t} J y\right)=\left(x_{j}^{t}, y_{j}^{t}\right)$. 


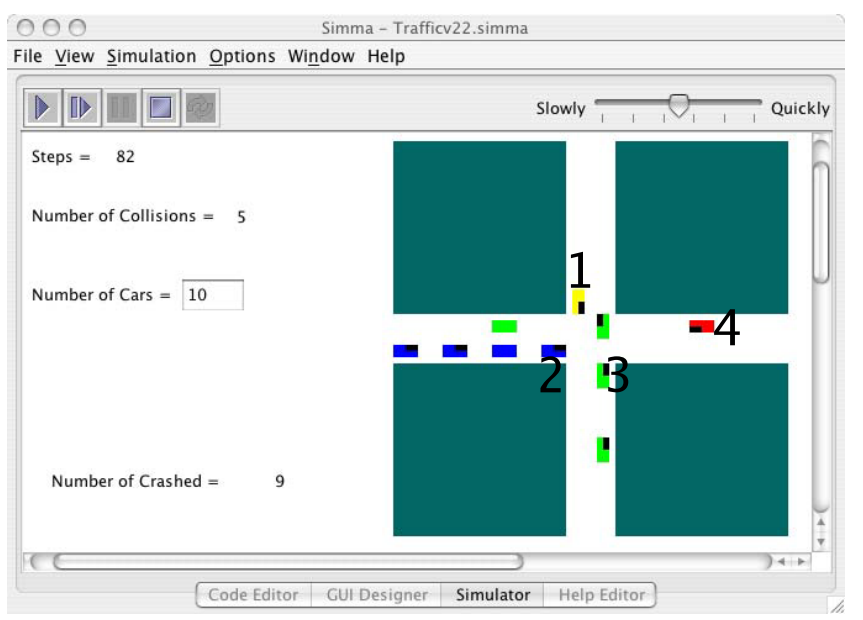

Fig. 3. Priority to give way to the right (Simma tool screenshot)

Table 1. Right priority norm

\begin{tabular}{|l|l|}
\hline Action & $\begin{array}{l}\text { in }\left(a_{i}, J_{B E}, t-1\right) \wedge \\
\text { in }\left(a_{i},\left(x_{i}^{t-1}+h_{i x}^{t-1}, y_{i}^{t-1}+h_{i y}^{t-1}\right), t\right) \wedge \\
\text { indicator }\left(a_{i}, \text { right }, t-1\right)\end{array}$ \\
\hline Pre-conditions & right $\left(a_{i}, a_{j}, t-1\right)$ \\
\hline Consequence & points points $_{i}^{t}-$ fine \\
\hline
\end{tabular}

Other norms, such as deadlock avoidance or junction blocking prevention have been considered and implemented. Nevertheless, due to the lack of space, we cannot detail them.

\subsection{Experimental Settings and Design}

As a proof of concept of our proposal in section 2.1, we have designed an experimental setting that implements the traffic case study. In this preliminary experiment we consider a single normative goal (num_collisions) and the right priority norm in table 1, which is parameterized by its fine (i.e., points to subtract to the car falling to follow the norm).

The 2-road junction traffic model has been developed with Simma [12, a graphical MAS simulation tool shown in Figure 3, in such way that both environment and agents can be easily changed. In our experimental settings, we have 


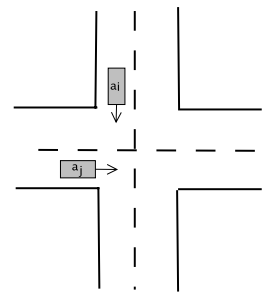

Fig. 4. Priority to give way to the right

modeled the environment as a $16 \times 16$ grid where both crossing roads have 2 lanes with opposite directions. Additionally, the environment is populated with 10 cars, having 40 points each.

Our institution can observe the agents properties for each tick and can keep a record of them in order to refer to past ticks. In fact, the institution usually determines traffic offenses by analyzing agent actions along time. Agent actions are observed through consecutive car positions and indicators (notice that the usage of indicators is compulsory for cars in this problem set up). During our discrete event simulation, the institution replaces those cars running out of points by new cars, so that the cars' population is kept constant. Cars follow random trajectories at a constant 1-cell/tick speed and they collision if two or more cars run into the same cell. In that case, the involved cars do remain for two ticks in that cell before they can start following a new trajectory.

Cars correspond to agents without learning skills. They just move based on their trajectories and institutional norms. Agents have local information about their environment (i.e., grid surrounding cells) and know whether their next moves will violate a norm and what fine will be thus applied. Agents decide whether to comply with a norm based on three parameters: $\langle$ fulfill_prob, high_punishment, inc_prob $\rangle$. Being fulfill_prob $\in[0,1]$ the probability of complying with norms that is initially assigned to each agent, high_punishment $\in \mathbb{N}$ the fine threshold that causes an agent to consider a fine to be high enough to reconsider the norm compliance, and inc_prob $\in[0,1]$ the probability increment that is added to fulfill_prob when the fine threshold is surpassed by the norm being violated. In summary, agents decide whether they keep moving regardless of violated norms or they stop in order to comply with norms based on a probability that is computed as: final_prob $=$ fulfill_prob $+i n c \_p r o b$ when fine $>$ high_punishment.

Our goal is to adapt norms to agent behaviors by applying Genetic Algorithms (GA) 1 to accomplish the institutional goal, to minimize the total number of collisions. We propose learn the norms by different agent populations behavior by simulation. Once specified what are the different agent populations behavior, a genetic algorithm is running by each population of agents. We use 10 individuals in each step of the genetic algorithm, where each individual is a set of parameters. Therefore, norm adaptation is implemented as a learning process

\footnotetext{
${ }^{1}$ We use GAlib [13], a C++ library of genetic algorithm components.
} 
of the "best" norm parameters. To evaluate an individual we run 10 times the simulator with the set of parameters of the individual. The simulator run the AEI model explained above during 5000 ticks. Thus, norm quality is given by a fitness function that considers the number of collisions, which is computed as an average of 10 different 5000-tick-long simulations for each model setting.

\section{Results}

From the experimental settings specified above, we have run experiments for three different agent populations. These populations are characterized by their norm compliance parameters, being fulfill_prob $=0.5$ and $i n c \_p r o b=0.4$ for the three of them whereas high_punishment varies from 5 for the first, to 10 for the second, up to 14 for the third (see table 2).

Since the right priority norm contributes to reduce accidents, our AEI must learn how to vary its fine parameter to increase its persuasiveness for agents, and eventually, to accomplish the normative goal of minimizing the total number of collisions. As to shows table 2, our experiments have resulted in that our AEI learned a fine of 14,12 , and 15 for each respective population. In all three cases, the learned fine is larger than the population's high_punishment value, and therefore, the goal is successfully reached 2 . In this manner, we can state the AEI success in learning the norms that better accomplish its goal.

Table 2. Learning results for three different agent populations

\begin{tabular}{|l|c|c|c|}
\hline Parameters & population1 & population2 & population3 \\
\hline fulfill_prob & 0.5 & 0.5 & 0.5 \\
\hline high_punishment & 5 & 10 & 14 \\
\hline inc_prob & 0.4 & 0.4 & 0.4 \\
\hline \hline Learned fine & $\mathbf{1 4}$ & $\mathbf{1 2}$ & $\mathbf{1 5}$ \\
\hline
\end{tabular}

Next figure 5 gives some more detail about the performance of agent populations for different norm fine values. First chart compares the number of collisions per 100 ticks when the fine is 4 with the resulting number of collisions when it is 14 , which is the learnt value for agents with a high_punishment threshold equal to 5. Analogously, second and third charts compare results between value 4 and learnt values 12 and 15 respectively (which, again, are learnt when the corresponding agent populations have 10 and 14 threshold values). For all three cases, we can observe that the number of collisions for fine 4 keep above the ones for learnt fines. It is so both in average and along the curve that results from a simulation of 5000 ticks. As expected, the reason is that value 4 is smaller than the high_punishment values for all three agent populations. Additionally, we can also observe that the deviation in the number of collisions is smaller as well.

\footnotetext{
${ }^{2}$ Notice that, due to the agent's behavior, any fine value higher that the population high_punishment value will be equally successful.
} 
Population 1: $<0.5,5,0.4>$

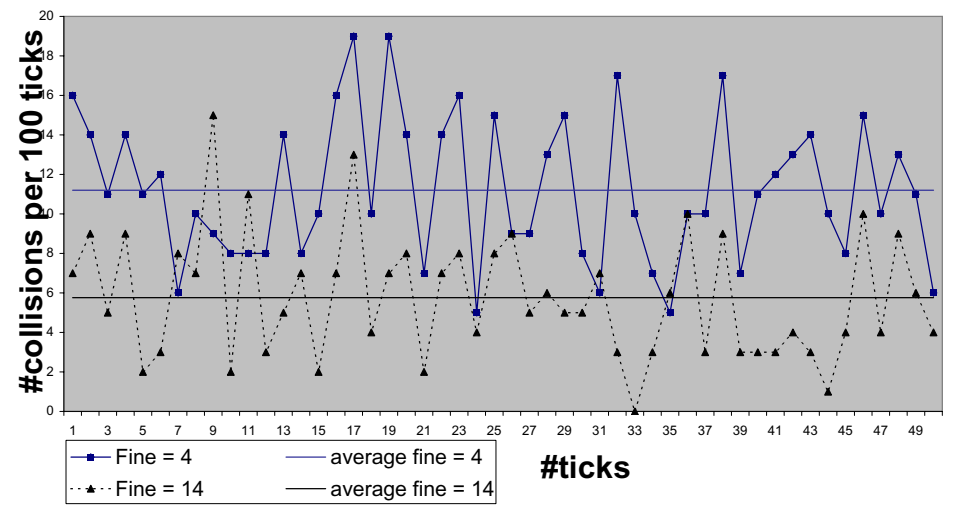

Population 2: $\langle 0.5,10,0.4\rangle$

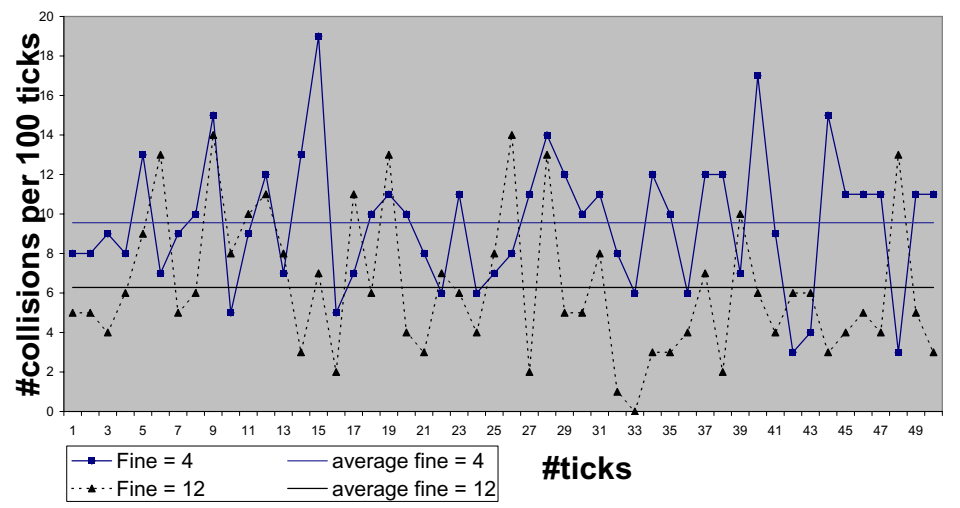

Population 3: $<0.5,14,0.4>$

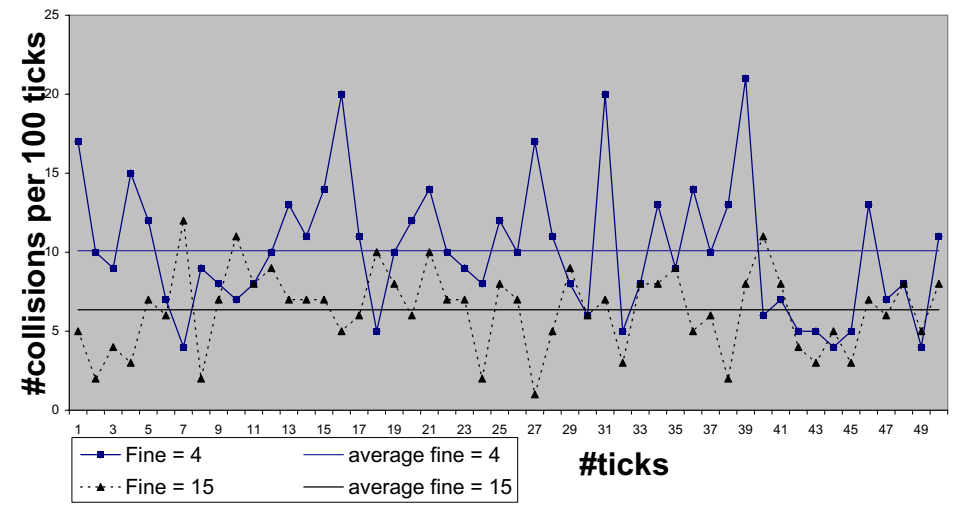

Fig. 5. Number of collisions per 100 ticks along a 5000-tick simulation 


\section{Discussion and Future Work}

Within the area of Multi-Agent Systems, adaptation has been usually envisioned as an agent capability. In this manner, works such as the one by Excelente-Toledo and Jennings 14 propose a decision making framework that enables agents to dynamically select the coordination mechanism that is most appropriate to their circumstances. Hübner et al. 15] propose a model for controlling adaptation by using the MOISE+ organization model. Agents in this model adapt their MAS organization to both environmental changes and their own goals. In [16] Gasser and Ishida presented a general distributed problem-solving model which can reorganize its architecture, in [17] Ishida and Yokoo introduce two new reorganization primitives that change the population of agents and the distribution of knowledge in an organization; and Horling et al. 18, propose an approach where the members adapt their own organizational structures at runtime. Norman et al. [19, within the CONOISE project, propose an agent-based model for dynamic formation of virtual organisations. However all these approaches are based on agent capabilities instead of on the use of norms. On the other hand, it has been long stated [20] that agents working in a common society need norms to avoid and solve conflicts, make agreements, reduce complexity, or to achieve a social order. Both approaches -i.e. adaptation and norms- have been considered together by Lopez-y-Lopez et al. 21, where agents can adapt to norm-based systems and they can even autonomously decide its commitment to obey norms in order to achieve associated institutional goals. This adaptation from the point of view of agents in these related works is the most remarkable difference with the approach presented in this paper, which focuses on adapting the institution -that is, the authority issuing norms- rather than adapting the agents. Institution adaptation is accomplished by changing norms autonomously (as opposite to the work by Hoogendoorn et al. [22, which is based on design considerations). Therefore, we do not select norms at design stages as it is done by Fitoussi and Tennenholtz [23, who do it so by proposing the notions of minimality and simplicity as selecting criteria. They study two basic settings, which include Automated-Guided-Vehicles (AGV) with traffic laws, by assuming an environment that consists of (two) agents and a set of strategies available to (each of) them. From this set, agents devise the appropriate ones in order to reach their assigned goals without violating social laws, which must be respected.

Regarding the traffic domain, MAS has been previously applied to it [1] 24] 25]. But traffic has been also widely studied outside the scope of MAS, for example, the preliminary work by [26] used Strongly Typed Genetic Programming (STGP) to controll the timings of traffic signals within a network of orthogonal intersections. Their evaluation function computed the overall delay.

This paper presents AEI as an extension of EIs with autonomic capabilities. In order to test our model, we have implemented a traffic AEI case study, where the AEI learns a traffic norm in order to fulfill its goals. Preliminary results in this paper provide soundness to our AEI approach. Recently, we have extended the AEI definition 27] in order to include a performative structure transition function in order to adapt performative structures. We are also currently performing 
the same experiments with other norms and with more goals. As future work, and since this basically represents a centralized scenario, we plan to develop a more complex traffic network, allowing us to propose a decentralized approach where different areas (i.e., junctions) are regulated by different institutions. Additionally, we are interested in studying how institutional norms and agent strategies may co-evolve. Nevertheless, this will require to extend the agents so that they become able to adapt to institutional changes. Nevertheless, we plan to extend both our traffic model and the institutional adaptation capabilities so that the AEI will not only learn the most appropriate norms for a given agent population, but it will be able to adapt to any change in the population.

\section{Acknowledgements}

This work was partially funded by the Spanish Science and Technology Ministry as part of the Web-i-2 project (TIC-2003-08763-C02-01) and by the Spanish Education and Science Ministry as part of the TIN2006-15662-C02-01 and the 2006-5-0I-099 projects. The first author enjoys an FPI grant (BES-2004-4335) from the Spanish Education and Science Ministry.

\section{References}

1. Luck, M., McBurney, P., Shehory, O., Willmott, S.: Agentlink Roadmap. Agenlink.org (2005)

2. Kephart, J.O., Chess, D.M.: The vision of autonomic computing. IEEE Computer 36(1), 41-50 (2003)

3. Esteva, M.: Electronic Institutions: from specification to development. IIIA PhD Monography, vol. 19 (2003)

4. Jennings, N.R., Sycara, K., Wooldridge, M.: A roadmap of agent research and development. Autonomous Agents and Multi-agent Systems 1, 275-306 (1998)

5. Noriega, P.: Agent-Mediated Auctions: The Fishmarket Metaphor. IIIA Phd Monography, vol. 8 (1997)

6. Arcos, J.L., Esteva, M., Noriega, P., Rodríguez-Aguilar, J.A., Sierra, C.: Engineering open environments with electronic institutions. Engineering Applications of Artificial Intelligence 18, 191-204 (2005)

7. North, D.: Institutions, Institutional Change and Economics Perfomance. Cambridge U. P., Cambridge (1990)

8. Etzioni, A.: Modern Organizations. Prentice-Hall, Englewood Cliffs, NJ (1964)

9. Gelfond, M., Lifschitz, V.: Representing action and change by logic programs. Journal of Logic Programming 17, 301-321 (1993)

10. Yang, Q.: A Simulation Laboratory for Evaluation of Dynamic Traffic Management Systems. PhD thesis, MIT (1997)

11. Luke, S., Cioffi-Revilla, C., Panait, L., Sullivan, K.: Mason: A new multi-agent simulation toolkit. In: Proceedings of the 2004 SwarmFest Workshop, p. 8 (2004)

12. López-Sánchez, M., Noria, X., Rodríguez-Aguilar, J.A., Gilbert, N.: Multi-agent based simulation of news digital markets. International Journal of Computer Science and Applications 2(1), 7-14 (2005) 
13. Wall, M.: GAlib, A C++ Library of Genetic Algorithm Components. Massachusetts Institute of Technology (MIT), http://lancet.mit.edu/ga/

14. Excelente-Toledo, C.B., Jennings, N.R.: The dynamic selection of coordination mechanisms. Autonomous Agents and Multi-Agent Systems 9(1-2), 55-85 (2004)

15. Hübner, J.F., Sichman, J.S., Boissier, O.: Using the Moise+ for a cooperative framework of mas reorganisation. In: Bazzan, A.L.C., Labidi, S. (eds.) SBIA 2004. LNCS (LNAI), vol. 3171, pp. 506-515. Springer, Heidelberg (2004)

16. Gasser, L., Ishida, T.: A dynamic organizational architecture for adaptive problem solving. In: Proc. of AAAI-91, Anaheim, CA, pp. 185-190 (1991)

17. Ishida, T., Yokoo, M.: Organization self-design of distributed production systems. IEEE Trans. Knowl. Data Eng. 4(2), 123-134 (1992)

18. Horling, B., Benyo, B., Lesser, V.: Using Self-Diagnosis to Adapt Organizational Structures. In: Proceedings of the 5th International Conference on Autonomous Agents, pp. 529-536 (2001)

19. Norman, T.J., Preece, A., Chalmers, S., Jennings, N.R., Luck, M., Dang, V.D., Nguyen, T.D., Deora, V., Shao, J., Gray, W.A., Fiddian, N.J.: Conoise: Agentbased formation of virtual organisations. In: Gedeon, T.D., Fung, L.C.C. (eds.) AI 2003. LNCS (LNAI), vol. 2903, pp. 353-366. Springer, Heidelberg (2003)

20. Conte, R., Falcone, R., Sartor, G.: Agents and norms: How to fill the gap? Artificial Intelligence and Law 7, 1-15 (1999)

21. López-López, F., Luck, M., d'Inverno, M.: Constraining autonomy through norms. In: Alonso, E., Kudenko, D., Kazakov, D. (eds.) AAMAS '02: Proceedings of the 1st international joint conference on Autonomous agents and multiagent systems, pp. 674-681. ACM Press, New York, USA (2003)

22. Hoogendoorn, M., Jonker, C., Treur, J.: Redesign of organizations as a basis for organizational change. LNCS, vol. 4386, pp. 51-71. Springer, Heidelberg (2007)

23. Fitoussi, D., Tennenholtz, M.: Choosing social laws for multi-agent systems: Minimality and simplicity. Artificial Intelligence 119(1-2), 61-101 (2000)

24. Dresner, K., Stone, P.: Multiagent traffic management: An improved intersection control mechanism. In: The 4th International Joint Conference on Autonomous Agents and Multiagent Systems, pp. 471-477. ACM Press, New York (2005)

25. Doniec, A., Espié, S., Mandiau, R., Piechowiak, S.: Dealing with multi-agent coordination by anticipation: Application to the traffic simulation at junctions. In: EUMAS, pp. 478-479 (2005)

26. Montana, D.J., Czerwinski, S.: Evolving control laws for a network of traffic signals. In: Genetic Programming 1996: Proceedings of the 1st Annual Conference, Stanford University, CA, USA, pp. 333-338. MIT Press, Cambridge (1996)

27. Bou, E., López-Sánchez, M., Rodríguez-Aguilar, J.A.: Adaptation of Autonomic Electronic Institutions through norms and institutional agents. In: 7th Annual International Workshop Engineering Societies in the Agents World (ESAW'06), pp. 137-152 (2006) 